\title{
A call to mandate patient access to personal primary care medical records across Canada
}

\author{
Iris Gorfinkel MD, Joel Lexchin MD
}

Cite as: CMAJ 2018 July 23;190:E869-70. doi: 10.1503/cmaj.180522

E ighty-five percent of family physicians use an electronic medical record. ${ }^{1}$ Yet, despite this, clinicians other than the patients' family physicians still struggle to access medical information for patients they encounter. This is exacerbated by patients' lack of knowledge of their own medical conditions and treatments. Patients are not enabled to provide important background information to all the practitioners who manage their care.

Lack of background information has been shown to result in needless retesting, heightened prescription errors, compromised patient care and increased overall costs. ${ }^{2}$ The problem could easily be remedied if patients had access to their own primary care records.

A 1993 Supreme Court of Canada decision made it clear that the information in the medical record belongs to the patient, stating, "Patients have a right to see the content of their record at any time and for any reasons." ${ }^{3}$ Yet patients face substantial impediments to accessing their primary care charts, including having to fill in authorization forms, pay transfer fees and deal with limited primary care office hours.

Records accessible to patients need not be all inclusive, but should, at a minimum, include the primary care physician's cumulative patient profile. This profile is a functional, concise document summarizing a patient's social, family, medical and surgical history; substance use; current medications; previous drug intolerances; and drug allergies. Consultation notes from specialists, radiology reports and laboratory results should also be available to patients so that all this information can be shared with other people involved in their treatment.

Efforts are ongoing to make patients' information securely available to multiple practitioners. One such effort is the Dossier santé Québec. It is not accessible to patients, but gives physicians and pharmacists access to prescription medications, laboratory test results and medical imaging results. ${ }^{3}$ Another example of record-sharing, also inaccessible to patients themselves, is ConnectingOntario, which provides access to records for more than 117000 clinicians in Ontario. ${ }^{4}$

Canadian health care institutions are also making a start on ensuring patient access to records. MyChart is a service available in 130 countries and currently available in seven hospitals in Ontario. MyChart allows patients to view their hospital records and to grant access to caregivers and clinicians (www.mychart. $\mathrm{ca} /$ ). There is a second patient portal through MyUHN at the Uni-

\section{KEY POINTS}

- Most patients in Canada do not have access to their primary care records.

- Provincial governments should mandate patient portals of access to electronic medical records.

- Having access to their health information affirms and validates that patients can act to ensure their own best health.

versity Health Network (comprising Toronto General Hospital, Toronto Western Hospital, Toronto Rehabilitation Institute and Princess Margaret Hospital), which allows patients access to appointments, results, reports and clinical documentation (www. uhn.ca/corporate/News/Pages/myUHN_patient_portal_patient_and _doctor_tell_how_made_difference.aspx). Private laboratories offer patients in Quebec, Ontario, British Columbia and Saskatchewan direct access to laboratory results. Alberta Netcare, a system expected to be in place in 2018 or 2019 , will enable facilities to submit key health information that forms an integrated patient record; this will be accessible by registered physicians (www.albertanetcare. ca/WhatIsAnEHR.htm).

Each of these initiatives represents an important step toward getting information into the hands of patients. However, more needs to be done to ensure easy, secure access for patients to their medical information across Canada, because transparency in clinical records supports patient-centred care.

Patients who believe that they play an important role in managing their own health are more likely to take affirmative action to manage their health, as measured by agreement with statements like "I know how to prevent problems with my health" and "I am confident that I can tell a doctor my concerns, even when he or she does not ask." ${ }^{5}$ When patients are more actively engaged in their own health, overall costs to the health system for the treatment of hyperlipidemia, hypertension and asthma are lower, and visits to emergency departments and admissions to hospital are fewer. ${ }^{5}$ When patients do not understand their treatments, they are less likely to take prescriptions, are less inclined to follow up with care and have greater morbidity. ${ }^{6}$

Offering patients portals of access to primary care records supports patient engagement and encourages them to participate in 
the management of their own health care. Having access to their health information empowers patients to be able to update primary care physicians or nurse practitioners should they encounter inaccuracies in their records, supporting vital communication between primary care providers and other health care practitioners. It affirms and validates that patients can act to ensure their own best health.

Sweden has led the way in offering patients universal access to medical records. All Swedish hospitals, primary care centres and psychiatric facilities use electronic medical records, and patients can log in to see notes from their health care professionals, a list of prescribed medications, test results, warnings, diagnoses, maternity care records, referrals, vaccinations and a log of everyone who has accessed their record. Research shows that $89 \%$ of patients who were surveyed strongly agreed that access to health records was positive, and $43 \%$ of the population had logged in through the national patient portal as of September 2017. ${ }^{7}$

There are potential barriers to implementing patient portals at many system levels. Primary care physicians may have justifiable concerns about the impact such a change will have on their workload. However, research examining the impact that patient portals have on physician workload is conflicting, showing both declines and increases in workload, depending on the study. ${ }^{8}$ Federal and provincial ministries of health will need to fund electronic medical record providers to develop, integrate and maintain patient portals. Costs associated with implementation could be a major deterrent. ${ }^{9}$ From the patients' perspective, there are also barriers to be overcome. There may be concerns regarding the security of patient-controlled portals. To counter that concern, the Canadian Medical Protective Agency suggests that doctors discuss in advance with patients the amount of medical information the portal should deliver (www.cmpa-acpm.ca/serve/docs/ela/goodpracticesguide/ pages/communication/Documentation/who_owns_the_medical_ record-e.html). Patients could log in with a private username and password to access and share their cumulative patient profile, and consult notes, laboratory results and radiology reports. Ease of use may also be a concern for patients and providers who are not familiar with technology. Some will inevitably have difficulties with gaining access to their records and will require technical support. Records must also be easily understood by older adults, by those with lower educational levels and by socioeconomically deprived populations. ${ }^{10}$ Costs would be offset by better patient engagement and self-management, improved patient care, a reduction in the need for repeated testing and decreased errors in drug prescribing.

Despite the barriers, we believe that Canada's provincial governments should mandate patient portals of access to electronic medical records, as such a commitment to health information transparency would herald a new era in patient empowerment. Fully patient-centred care can begin only when patients are able to access their primary care records and share them with their physicians when most needed. Without this ability, patients and their families suffer needlessly, physicians are less effective and the costeffectiveness of our universal health care system is diminished. Harnessing the tremendous, untapped potential of patient engagement is a triple win for patients and their caregivers, for their treating physicians and for the health care system as a whole.

\section{References}

1. CMA physician workforce survey, 2017. National results by FP/GP or other specialist, gender, age, and province/territory. Ottawa: Canadian Medical Association; 2017. Available: http://cma.andornot.com/SurveyPDF/CMA_Survey_Workforce2017 _Q22_ElectronicRecords-e.pdf (accessed 2018 June 18).

2. Tharmalingam S, Hagens S, Zelmer J. The value of connected health information: perception of electronic health record users in Canada. BMC Med Inform Decis Mak 2016;16:93.

3. Dossier santé Québec. Gouvernement du Québec; 2018. Available: www.quebec.ca/ sante/vos-informations-de-sante/dossier-sante-quebec/ (accessed 2018 June 30).

4. ConnectingOntario ClinicalViewer. Toronto: eHealth Ontario; 2018. Available: www.ehealthontario.on.ca/for-healthcare-professionals/connectingontario (accessed 2018 June 30).

5. Hibbard JH, Greene J, Overton V. Patients with lower activation associated with higher costs; delivery systems should know their patients' "scores." Health Aff (Millwood) 2013;32:216-22.

6. Squier RW. A model of empathic understanding and adherence to treatment regimens in practitioner-patient relationships. Soc Sci Med 1990;30:325-39.

7. Hägglund M, Moll J, Åhlfeldt R-M, et al. Timing it right - patients' online access to their record notes in Sweden. Stud Health Technol Inform 2018;247:336-40.

8. Mold F, de Lusignan S. Patients' online access to their primary care electronic health records and linked online services: implications for research and practice. $J$ Pers Med 2015;5:452-69.

9. Kruse CS, Argueta DA, Lopez L, et al. Patient and provider attitudes toward the use of patient portals for the management of chronic disease: a systematic review. $J$ Med Internet Res 2015;17:e40.

10. Ware P, Bartlett $S$, Paré G, et al. Using ehealth technologies: interests, preferences, and concerns of older adults. Interact J Med Res 2017;6:e3.
Competing interests: As the principal investigator and founder of PrimeHealth Clinical Research, Iris Gorfinkel reports receiving grants for research from many major pharmaceutical companies during her participation in more than 50 clinical research trials. In addition, she has participated in speaking engagements for pharmaceutical companies. She has sat on the advisory board for GlaxoSmithKline's Shingrix vaccination. Joel Lexchin reports acting as a paid consultant on projects for the US Agency for Healthcare Research and Quality, the Gordon and Betty Moore Foundation, and the Ontario Strategy for Patient-Oriented Research Support Unit and the St. Michael's Hospital Foundation. He also reports receiving payments for participating in a panel that discussed a pharmacare plan for Canada (Canadian Institute, a for-profit organization), a panel at the American Diabetes Association and giving a talk at the Toronto Reference Library. He is currently a member of research groups that are receiving money from the Canadian Institutes of
Health Research and the Australian National Health and Medical Research Council. He is member of the Foundation Board of Health Action International and the Board of Canadian Doctors for Medicare.

This article has been peer reviewed.

Affiliations: PrimeHealth Clinical Research - Family Practice (Gorfinkel); School of Health Policy and Management (Lexchin), Faculty of Health, York University, Toronto, Ont.

Contributors: Iris Gorfinkel conceived of the idea for this work. Both authors contributed to the design of the work, drafted the manuscript, revised it critically for important intellectual content, gave final approval of the version to be published and agreed to be accountable for all aspects of the work.

Correspondence to: Iris Gorfinkel, i.gor@outlook.com 